\title{
Compactification on negatively curved manifolds
}

\section{Michael R. Douglas ${ }^{a, b}$ and Renata Kallosh ${ }^{c}$}

${ }^{a}$ Simons Center for Geometry and Physics, Stony Brook University, Stony Brook, NY 11794, U.S.A.

${ }^{b}$ I.H.E.S., Le Bois-Marie, 35 route de Chartres, 91440 Bures-sur-Yvette, France

${ }^{c}$ Department of Physics, Stanford University, Stanford, CA 94305, U.S.A.

E-mail: douglas@max2.physics.sunysb.edu, kallosh@stanford.edu

ABSTRACT: We show that string/M theory compactifications to maximally symmetric space-times using manifolds whose scalar curvature is everywhere negative, must have significant warping, large stringy corrections, or both.

KEYWORDS: Flux compactifications, dS vacua in string theory, Classical Theories of Gravity, Supergravity Models

ARXiv EPRINT: 1001.4008 


\section{Contents}

1 Introduction 1

2 Basic argument $\quad 3$

2.0.1 Magnetic $p$-form flux 4

2.0.2 Electric flux 5

2.1 Localized sources 6

$\begin{array}{lll}2.2 & \text { Total curvature, no warping case } & 7\end{array}$

2.3 Internal energy conditions 8

3 Stringy corrections, conformal and warp factor dependence 9

$\begin{array}{ll}3.1 & \text { Stringy corrections }\end{array}$

$\begin{array}{lll}3.2 & \text { Warp factor } & 10\end{array}$

3.3 Conformal factor 11

4 Discussion $\quad 12$

A Type IIA, IIB $d=10$ Supergravity Energy-Momentum Tensor $\quad 14$

\section{Introduction}

If our universe is described by string/M theory, there exist six or seven extra dimensions of space, not yet detected by experiment. This is possible if the extra dimensions take the form of a small, compact manifold $M$. There are many possible choices of $M$ and it is very interesting to find constraints on this choice, both internal consistency conditions, and those required to match the observations.

In this note we find constraints on the possibility of using a $k$-dimensional manifold $M^{k}$ with a metric of negative curvature. Mathematically, the characterization of a manifold with metric as having positive, zero, or negative curvature is one of the most important aspects of its geometry [1]. Familiar examples of the first two classes are the sphere $S^{k}$ and the torus $T^{k}$, while the higher genus Riemann surfaces provide an infinite set of negatively curved examples in two dimensions.

Examples of negatively curved manifolds with $k>2$ include hyperbolic manifolds, high degree hypersurfaces, nilmanifolds and solmanifolds in $k=3$, products of lower dimensional negatively curved manifolds, and others. While these may be less familiar, and since the curvature tensor has many components even the definition of "negative curvature" requires discussion, there are in a precise sense (as explained in [1]) many more negatively curved than non-negatively curved manifolds, just as for $k=2$. 
Using this trichotomy, we get a basic (highly oversimplified) classification of supergravity and string theory compactifications. The simplest case is zero curvature, here meaning Ricci flat. In this case, $M$ solves the vacuum Einstein equations, and we get a compactification to Minkowski space-time. This includes tori, Calabi-Yau manifolds, $G_{2}$ manifolds, and singular limits of these such as orbifolds. One can of course turn on more fields and vary the Ricci curvature away from zero, but the global features of the solution will not change, unless we leave the supergravity regime.

For many manifolds, no Ricci flat metric is believed to exist. In this case, one must try to solve the Einstein equation with a source of stress-energy, say from flux ( $p$-form gauge fields). In the simplest and by now very well-known solutions of this type, space-time is a direct product of anti de-Sitter space-time and a sphere. Thus, the internal manifold has positive curvature. This class of solution can be generalized to many other positive curvature manifolds, obtained by quotienting by a group action, blow-up, and various other constructions.

Both constructions lead to a wide variety of compactifications on $M$ with positive or zero Ricci curvature. On the other hand, there are few known families of solutions based on other types of $M$, with negative or mixed Ricci curvature. Indeed, at this writing, there are no such compactifications to maximally symmetric space-times (Minkowski, de Sitter or anti-de Sitter) for which the higher dimensional field configurations are known explicitly or at least proven to exist. Assuming this can be done, we would like to decide whether such solutions are common or else rare, relying on structures and coincidences which only hold in a few examples.

One particular reason to be interested in this is to find de Sitter solutions. It was noticed in [2] that the four dimensional curvature gets a significant positive contribution from the negative curvature of the internal space. This could serve as an important contribution to the uplifting of the string theory vacua which tend to be anti de-Sitter solutions, rather than de Sitter. Indeed, we will show, starting with ten dimensional Einstein equations, that the four dimensional curvature scalar $R_{4}$ is defined by the four dimensional energy-momentum tensor trace $T_{4}$ as well as by the six dimensional curvature scalar $R_{6}$

$$
R_{4}=-T_{4}-2 R_{6}
$$

Clearly, a negative curvature of the compactified manifold $R_{6}<0$ may, in principle, overcompensate the possible negative contribution from $T_{4}$ term and produce a de Sitter minimum with $R_{4}>0$. This feature of the negative curvature has allowed to present an explicit class of de Sitter vacua in massive type IIA supergravity [3] with local sources, which evade the no-go theorems discussed in $[4,5]$. The analogous situation concerns the no-go theorems for slow-roll inflation in [6]. The presence of the negative curvature in model [7] helps to provide a slow-roll inflationary model which makes an interesting prediction of a detectable level of gravity waves. See [8] for another recent work linking negative curvature and cosmology.

In this note, we study maximally symmetric supergravity solutions. There is a very simple "no-go" argument, which we give in section 2, that in the absence of warping, and if the stress-tensor satisfies an energy condition which holds for all the standard forms 
of matter (including the mass term of IIa supergravity), the internal manifold $M$ must have nonnegative scalar curvature at each point. While both conditions can be relaxed in string/M theory compactification, this simple argument provides a useful starting point for a more general discussion.

The argument is very similar to the no-go theorem of [9-12]. We identify the combination of the Einstein equations which determines the scalar curvature, and find an inequality satisfied by the source. However an important difference in the present case is that this imposes a condition at each point of $M$. As we discuss in section 2.1, most of the string/M theory terms which violate this inequality, such as orientifold planes, are localized. Thus, there will be many regions in $M$ in which they do not contribute, and thus they cannot by themselves evade this "no-go" result.

In section 3, we discuss ways to get negative curvature which could work within the regime described by supergravity with branes and stringy corrections. Of course, we know of string/M theory solutions which are not well described by supergravity, but since the concept of negative curvature (or even curvature) has not yet been defined for such solutions, it is too early to place them in the general scheme we are discussing.

In section 4, we comment on the possible implications of this for solutions which have been shown to exist using effective potential arguments.

\section{Basic argument}

We focus on string theory and $d=10$ supergravity with local sources. In M-theory and $d=11$ supergravity with local sources the results are analogous. Our assumptions are:

1. We assume that $10 \mathrm{~d}$ is a product of $6 \mathrm{~d}$ and $4 \mathrm{~d}$ and the $4 \mathrm{~d}$ space is symmetric, i.e. $T_{\mu \nu} \sim g_{\mu \nu}$ and form fields $F_{\mu \nu \rho \sigma m n \ldots}$ are proportional to $\epsilon_{\mu \nu \rho \sigma}$.

2. We consider the simplest case that the geometry is not warped: the function of $6 \mathrm{~d}$ $y$ coordinates denoted $\Delta^{-1}(y)$ in [11] and $\Omega^{2}(y)$ in [12] is constant, $\Delta(y) \sim \Omega(y) \sim$ const.

Starting from the Einstein equations,

$$
R_{M N}-\frac{1}{2} g_{M N} R=T_{M N}
$$

we pass to the trace reversed form,

$$
R_{M N}=T_{M N}-\frac{1}{8} g_{M N} T_{L}^{L}
$$

Here $L=0, \ldots, 9, m=1, \ldots, 6$ and $\mu=0, . ., 3$. We introduce the notation

$$
R_{4}=R_{\mu \nu} g^{\mu \nu}, \quad R_{6}=R_{m n} g^{m n}, \quad T_{4}=T_{\mu}^{\mu}, \quad T_{6}=T_{m}^{m}
$$

If there is no warping, so the metric is a direct product, the curvature $R_{4}$ is constant on $M$, and is zero, positive or negative for Minkowski, dS and AdS space-times respectively. 
By tracing eq. (2.2) we find that

$$
R_{4}=-\frac{1}{2} T_{6}+\frac{1}{2} T_{4}
$$

and

$$
R_{6}=+\frac{1}{4} T_{6}-\frac{3}{4} T_{4}
$$

Note that combining eq. (2.4) with eq. (2.5) multiplied by 2, we reproduce eq. (1.1). The first of these coincides with eq. (33) in [12] and (2.12) in [11] (taking account of their conventions for the curvature), comparing in both cases with the non-warped case. The statement that its right hand side is non-positive is called the strong energy condition $[9,10$, 13]. It is satisfied by all standard forms of matter except for negative potential energy, and "0-form" flux [3]. Thus, it is very easy to see that de Sitter space-time, with $R_{4}>0$, cannot be obtained without either sources which violate the strong energy condition, or warping.

With a little more work, one can show that warping does not affect this conclusion. Although it makes another contribution to eq. (2.4), after integrating the equation over $M$ with a suitable positive weight, the extra contribution vanishes.

Clearly we can apply the same reasoning to eq. (2.5). Generalizing 4 and 6 to arbitrary dimensions $d$ and $k$, the claim is

$$
0 \leq R_{k}=\frac{d-2}{d+k-2} T_{k}-\frac{k}{d+k-2} T_{d}
$$

Let us check that its right hand side is non-negative for the standard forms of matter, now including 0-form flux.

The only input we need from supergravity is that the stress-tensors for the form fields are quadratic in the field strengths, and positive. In general, this is not completely obvious, especially for the case of IIb supergravity, because of the presence of Chern-Simons terms and modified Bianchi identities. However there is a formalism in which it is obvious, the democratic version of $d=10$ supergravity $[14,15]$, as we review in an appendix.

\subsubsection{Magnetic $p$-form flux}

$$
T_{m n}^{m a g}=p F_{m . .} F_{n}^{. .}-\frac{1}{2} g_{m n} F_{p}^{2} \quad \Rightarrow T_{6}^{\text {magn-p-form }}=(p-3) F_{p}^{2}
$$

so

$$
T_{\mu \nu}^{m a g}=-\frac{1}{2} g_{\mu \nu} F_{p}^{2} \quad \Rightarrow T_{4}^{\text {magn-p-form }}=-2 F_{p}^{2}
$$

Evaluating our general eq. (2.4) and eq. (2.5) we find

$$
R_{4}^{\text {mag }}=-\frac{1}{2} T_{6}+\frac{1}{2} T_{4}=\frac{1-p}{2} F_{p}^{2}
$$

and

$$
R_{6}^{\text {mag }}=+\frac{1}{4} T_{6}-\frac{3}{4} T_{4}=\frac{p+3}{4} F_{p}^{2} \text {, }
$$


verifying the claim. In $d$ and $k$ dimensions, the second equation becomes

$$
R_{k}^{\text {mag }}=\frac{1}{d+k-2}\left((d-2) T_{k}-k T_{d}\right)=\frac{(d-2) p+k}{d+k-2} F_{p}^{2} .
$$

Another way to think about this result is to note that the $k$-dimensional trace of the Einstein equations is proportional to the variation of the action with respect to the $k$ dimensional conformal factor (say as defined below in eq. (3.8)). Integrating over $M$, one relates this to the variation of the effective potential with respect to the volume of $M$. Writing this volume in terms of a length scale $L$ as,

$$
\int_{M} \sqrt{g} \equiv L^{k}
$$

naively this is

$$
\begin{gathered}
L \frac{\partial}{\partial L} \int \sqrt{g} g^{i_{1} j_{1}} \cdots g^{i_{p} j_{p}} F_{i_{1} \ldots i_{p}} F_{j_{1} \ldots j_{p}} \\
\sim(k-2 p) L^{k-2 p}
\end{gathered}
$$

as in eq. (2.7). This term can make contributions of either sign to $R_{6}$.

However, there is an additional factor from the rescaling to go to $d$-dimensional Einstein frame. Using the results of [16], one can show that this is

$$
\sqrt{g^{(d)}}=u^{2} \sim L^{-k d /(d-2)},
$$

which corresponds precisely to the $T_{d}$ dependence in eq. (2.11).

\subsubsection{Electric flux}

It is not hard to see that an electric contribution is dual to a magnetic flux, with $p$ replaced by $10-p=\tilde{p}$, for example,

$$
R_{6}^{e l-p-f o r m}=\frac{13-p}{4} \tilde{f}_{p}^{2}=\frac{\tilde{p}+3}{4} \tilde{f}_{10-\tilde{p}}^{2} \quad \Leftrightarrow \quad R_{6}^{\text {magn }-p-\text { form }}=\frac{p+3}{4} F_{p}^{2}
$$

Let us consider an electric form field with $p \geq 4$, with 4 legs in space-time and the rest in 6, i.e. for $F_{\mu \nu \rho \sigma m_{1} \ldots m_{p-4}}=i e \epsilon_{\mu \nu \rho \sigma} f_{m_{1} \ldots m_{p-4}}$ as shown in [11] we get

$$
\begin{gathered}
T_{m n}^{e l-p-f o r m}=-p(p-1)(p-2)(p-3)\left[(p-4) f_{m q_{1} \ldots q_{p-5}} f_{n}^{q_{1} \ldots q_{p-5}}-\frac{1}{2} g_{m n} f_{q_{1} \ldots q_{p-4}} f^{q_{1} \ldots q_{p-4}}\right] \\
T_{\mu \nu}^{e l-p-f o r m}=-\frac{1}{2} g_{\mu \nu} \tilde{f}_{p}^{2}
\end{gathered}
$$

where

$$
p(p-1)(p-2)(p-3) f_{q_{1} \ldots q_{p-4}} f^{q_{1} \ldots q_{p-4}} \equiv \tilde{f}_{p}^{2}
$$

and

$$
\begin{aligned}
& T_{6}^{e l-p-\text { form }}=(7-p) \tilde{f}_{p}^{2} \\
& T_{4}^{e l-p-\text { form }}=-2 \tilde{f}_{p}^{2}
\end{aligned}
$$

After substituting $p \rightarrow 10-p$, these agree with eqs. (2.7) and (2.8). The same is true for the linear combinations $R_{4}$ and $R_{6}$. 


\subsection{Localized sources}

Following [17], consider a p-brane wrapped on a (p-3)-cycle $\Sigma$

$$
S_{l o c}=-T_{p} \int_{R^{4} \times \Sigma} d^{p+1} \xi \sqrt{-\tilde{g}}+\mu_{p} \int_{R^{4} \times \Sigma} C_{p+1}
$$

where $T_{p}=\left|\mu_{p}\right| e^{p-3 \phi / 4}$ and $g$ is the determinant of the metric induced on the brane, $\tilde{g}_{\alpha \beta}=\frac{\partial X^{M}}{\partial \xi_{\alpha}} \frac{\partial X^{N}}{\partial \xi_{\beta}} g_{M N}$. When the brane is embedded into a 4 space-time dimensions and p-3 internal dimensions, i.e. $X^{\mu}=\xi^{\mu}$ and $X^{i}=\xi^{i}, i=1, \ldots p-3$, the variation of the Born-Infeld action leads to

$$
T_{\mu \nu}^{l o c}=-T_{p} g_{\mu \nu} \delta(\Sigma), \quad T_{m n}^{l o c}=-T_{p} \Pi_{m n}^{\Sigma} \delta(\Sigma), \quad \Pi_{m n}^{\Sigma} g^{m n}=p-3
$$

Here $\delta(\Sigma)$ means that the energy-momentum tensor from local sources is concentrated only at the (p-3)-cycle $\Sigma$. The projector $\Pi_{m n}^{\Sigma}$ from the 6 d space into a (p-3)-cycle shows that the brane action does not depend on the components of the metric in the direction orthogonal to the cycle, $i^{\prime}=(p-4), \ldots, 6$.

So we see that

$$
\begin{gathered}
T_{4}^{l o c}=-4 T_{p} \delta(\Sigma) \quad T_{6}^{l o c}=-T_{p}(p-3) \delta(\Sigma) \\
R_{4}^{l o c}=-\frac{1}{2} T_{6}+\frac{1}{2} T_{4}=\frac{p-7}{2} T_{p} \delta(\Sigma)
\end{gathered}
$$

and

$$
R_{6}^{l o c}=+\frac{1}{4} T_{6}-\frac{3}{4} T_{4}=\frac{15-p}{4} T_{p} \delta(\Sigma)
$$

We again find that these sources satisfy the expected inequalities, as long as the brane tension $T_{p}$ is positive. Following the discussion at the end of section 2.0.1, this will be true for any source with positive energy which falls off as the volume of $M$ goes to infinity. As pointed out in [18], this falloff would fail to hold only for an energy density which grows at least as fast as the volume, which seems highly unphysical. Thus the only way around the inequality is to use sources with $T_{p}<0$.

Of course, string theory contains such sources, for example orientifolds. Can they change the conclusion? As we noted in the introduction, the inequality eq. (2.6) must hold at every point in the internal manifold $M$. On the other hand, most branes and orientifolds are lower dimensional objects, and thus contribute only on submanifolds of $M$. Of course there is some sense in which these contributions are spread out over the string and Planck scales, but to analyze the solutions in detail so close to the source, we must leave the supergravity approximation, instead relying on open strings, gauge theory, or some other description. To work purely within supergravity, one normally one treats branes as delta function sources; we see no reason this approximation should be wrong in general (of course there might be subtleties in particular cases). Thus, even including branes and orientifolds supported on lower dimensional cycles, the inequality eq. (2.6) still leads to significant constraints, and rules out solutions with scalar curvature everywhere negative. 
In principle, the O9 might provide a counterexample, but anomaly cancellation will force there to be sufficiently many D9 branes to cancel this source.

Another idea sometimes called upon in this context is to spread out or "smear" an orientifold or brane source [19-21]. It was applied in the context of the IIA flux vacua $[4,5]$ of massive 10d supergravity [3] with local sources.

The idea is to replace a localized source by a smooth source with support throughout $M$, whose integral over $M$ is the same as the original source. While this certainly simplifies the task of finding a solution, and would avoid the issue we are describing, in the context of string compactification to dimensions $d>2$, it is not justified. First, in classical (genus zero) string theory, the location of a brane or orientifold plane is a modulus, which must be fixed to define the theory. Of course, in the full quantum theory one can propose a wave function which is delocalized in the moduli, and in this way define states in which the expectation value of the metric and other fields is smeared in this way. However, in dimensions $d>2$ this amounts to combining different superselection sectors, and such an expectation value is not physical; in a given superselection sector the expectation value of a brane or orientifold position will take a fixed value. This is not particular to string/M theory but is a general feature of quantum scalar fields in dimensions $d>2$.

Smearing is valid when constructing solitonic particle or string solutions, because their wave functions can superpose different values of the zero modes. Even in $d>2$, the question of whether smeared solutions exist is not without interest, as if they do not, one expects that no localized solution will exist either. However it is not really an answer to the issue we are raising.

\subsection{Total curvature, no warping case}

Combining the previous results, the conclusion is that

$$
R_{4}=-\frac{p-1}{2} F_{p}^{2}+\frac{p-9}{2} \tilde{f}_{p}^{2}+\frac{p-7}{2} T_{p} \delta(\Sigma)
$$

and

$$
R_{6}=\frac{p+3}{4} F_{p}^{2}+\frac{13-p}{4} \tilde{f}_{p}^{2}+\frac{15-p}{4} T_{p} \delta(\Sigma)
$$

The second term in each eq. is valid only for $p \geq 4$, so it is negative in $R_{4}$ and positive in $R_{6}$. In the third term $p \geq 3$ and the sign of $T_{p}$ is positive for the $\mathrm{D}$-p-branes and negative for the O-p-planes. It is nice to see that in both equations the duality condition with replacement of $p$ into $10-p$ and electric into magnetic fluxes, works in agreement with the generic condition given in eq. (A.6). Namely, in eq. (2.26) as well as in eq. (2.27)

$$
p \rightarrow 10-p, \quad F_{p}^{2} \rightarrow \tilde{f}_{p}^{2}
$$

is a symmetry of the flux contributions to the corresponding curvatures.

It may be also useful to provide the energy-momentum tensor sub-traces.

$$
T_{4}=-2 F_{p}^{2}-2 \tilde{f}_{p}^{2}-4 T_{p} \delta(\Sigma)
$$


and

$$
T_{6}=(p-3) F_{p}^{2}+(7-p) \tilde{f}_{p}^{2}+(3-p) T_{p} \delta(\Sigma)
$$

One can verify that $R=R_{4}+R_{6}$ and $T=T_{4}+T_{6}$ are related to each other by a factor $-4 R=T$ for each of the 3 contributions.

By looking at eq. (2.27) we see that fluxes always give positive contributions. While local sources may give a negative contribution, their negative energy is localized on cycles on which the branes are wrapped. Thus, using the sources discussed so far, there can be no solution of the $6 \mathrm{~d}$ Einstein equations with everywhere negative $6 \mathrm{~d}$ curvature.

\subsection{Internal energy conditions}

The results we just obtained suggest that we introduce a new "internal energy condition" (or IEC),

$$
T_{k} \geq \frac{k}{d-2} T_{d}
$$

which looks very similar to the strong energy condition (SEC),

$$
T_{k} \geq \frac{k-2}{d} T_{d}
$$

Their relationship depends on the sign of $T_{d}$. Since $T_{d}=-d \Lambda_{\text {matter }}=-d \rho$, this will normally be negative, in which case the IEC follows from the SEC and is strictly weaker.

We could also consider a "strong internal energy condition," which would guarantee that $R_{i j}$ be non-negative definite. ${ }^{1}$ Everywhere non-negative Ricci curvature is a far stronger geometric constraint than non-negative scalar curvature, ruling out the vast majority of manifolds [1]. This would hold if

$$
T_{i j} e^{i} e^{j} \geq \frac{1}{D-2}\left(T_{d}+T_{k}\right)|e|^{2} \quad \forall e \in T M .
$$

Is this ever violated? For magnetic fluxes, this becomes

$$
\left|e^{m} F_{m . .}\right|^{2} \geq \frac{p-1}{p(k+d-2)} F_{p}^{2}|e|^{2} \quad \forall e \in T M .
$$

For several fluxes, one would have a single condition obtained by adding the left and right hand sides separately. Note also that under $p \rightarrow k-p$ and $F \rightarrow *^{(k)} F$, the condition changes, but in a rather minor way; $(p-1) / p$ becomes $(k-p-1) /(k-p)$.

Intuitively, this condition will hold at a point if the flux is not too anisotropic, meaning that the ratio between flux in different planes stays below $(k+d-2)$. It is clearly true for $p=k$. And, since the coefficient on the right hand side is small, one might expect it to "usually" hold. But there are counterexamples, with the simplest perhaps being the supergravity solutions of [12]. These are obtained by compactifying some of the spatial AdS dimensions, and are not maximally symmetric in the remaining space-time dimensions, but they illustrate the possibility.

\footnotetext{
${ }^{1}$ This idea was discussed in passing in [22].
} 
For $p=2$ and $k-p=2$, which are the cases of most interest in IIa compactification with $k=6$, one can understand this condition using a change of basis to put $F$ or $* F$ in canonical form, which is $F_{2 n-1,2 n}=-F_{2 n, 2 n-1}$, all other components zero. In this case eq. (2.34) becomes

$$
F_{2 n-1,2 n}^{2} \geq \frac{p-1}{p(k+d-2)} \sum_{n} F_{2 n-1,2 n}^{2} .
$$

While clearly this can be violated, doing so requires the flux to be quite anisotropic.

\section{Stringy corrections, conformal and warp factor dependence}

Eq. (2.27) shows that almost all sources to $R_{6}$ are positive; the only negative sources in IIa compactification in the supergravity limit are the O6 planes, which are localized. Thus we cannot find solutions with everywhere negative scalar curvature, as is the case for the nilmanifolds used in [2].

There are several ways one can try to get around this in string/M theory. One is to call upon higher derivative terms or quantum corrections. Another is to vary the metric. Although this possibility is too difficult to analyze in generality, one can make fairly simple statements about the dependence on the warp and conformal factors.

\subsection{Stringy corrections}

The higher derivative terms of string/M theory, such as the $R^{4}$ term, see for example [23], clearly are not localized, and need not satisfy the positivity condition eq. (2.6). In fact, these terms can sometimes be shown to give negative contributions. For example, the $C^{3} \wedge R^{4}$ term of $\mathrm{M}$ theory, in the context of compactification on a Calabi-Yau fourfold as in [24], is topological, in fact the curvature term integrates to the Euler characteristic.

Of course, from the point of view of supergravity, these terms are corrections which are suppressed by factors such as the curvature of $M$ in string units. On the other hand, the fluxes are also quantized in string units, so that the leading stringy corrections can come in at the same order. This is familiar in the case of tadpole conditions, which relate topological numbers such as the number of units of flux, numbers of branes, and the Euler character of $M$.

In supersymmetric solutions, the Einstein equations we are discussing are usually partnered with tadpole conditions, making it natural to get Minkowski space-time. Without supersymmetry, this need not be the case. What sort of negative curvature can a term like this support? Let us write the $6 \mathrm{~d}$ trace of the Einstein equations schematically as

$$
R_{6} \sim-l_{s}^{6}\left(\operatorname{Riemann}_{6}\right)^{4}+T_{6}+\ldots
$$

The fourth order term is written this way to emphasize that it depends on all components of the Riemann tensor, and can be nonzero even if $M$ is Ricci flat. More generally, the magnitude of the Riemann tensor, and that of the scalar curvature $R_{6}$, are a priori not the same.

Assuming the negative $R^{4}$ term dominates, and estimating Riemann ${ }_{6} \sim 1 / L^{2}$ where $L$ is some characteristic size of $M$ (say the diameter), we can have $R_{6} \sim l_{s}^{6} / L^{8}$, with further 
corrections suppressed by $l_{s}^{2} / L^{2} \ll 1$. This is the same scaling as $p=3$-form flux. The string theory $R^{4}$ corrections of the type studied in [23] have been used in cosmology with large-volume flux compactifications in $[25,26]$. It remains to be seen if the analogous string type corrections may be useful for supporting the negative curvature of the the Nil 3 -manifolds of $[2,7]$.

\subsection{Warp factor}

Starting with a maximally symmetric space-time metric $\eta_{\mu \nu}$ and an internal metric $g_{i j}$, we consider the metric ansatz

$$
d s^{2} \equiv \tilde{g}_{M N} d x^{M} d x^{N}=e^{2 A(y)} \eta_{\mu \nu} d x^{\mu} d x^{\nu}+e^{2 B(y)} g_{i j} d y^{i} d y^{j},
$$

with two freely chosen functions $A$ and $B$. Simple computations done in [16] allow generalizing eq. (2.26) and eq. (2.27) to this case.

This modifies both equations, and the modified $R_{6}$ equation can be found in equation (5.2) in [16],

$$
\frac{k-2}{2} R^{(k)}=2(k-1) u^{-1} \nabla^{2} u+\frac{2(D-2)}{d}\left(u^{-1} \nabla u\right)^{2}-\frac{k}{2} u^{-4 / d} C-T^{(k)},
$$

where $u=e^{d A / 2}$. This has a sufficiently complicated dependence on the warp factor to counter the no-go theorem; in particular the $u^{-1} \nabla^{2} u$ term can easily be negative.

Of course, one must then ask whether there is some analog of the arguments in [9-12] which allowed rewriting these terms as a total derivative, thus getting a constraint on the integral, independent of warping. Thus, let us consider the most general integral constraint, obtained by integrating eq. (3.3) against a weight $u^{\alpha}$, and adding $\beta$ times the warp factor constraint (eq. (2.33) in [16]),

$$
-\frac{d-2}{2} R^{(d)} u^{1-4 / d}=-2(d-1) \nabla^{2} u+\left(\frac{d}{2} R^{(k)}+T^{(d)}\right) u,
$$

integrated against the weight $u^{\alpha-1}$. Integrating by parts and multiplying by 2 , this leads to

$$
\begin{aligned}
(k-2-\beta d) \int u^{\alpha} R^{(k)}= & \left(\frac{4(d+k-2)}{d}-4(\alpha-1)(k-1-\beta(d-1)) \int u^{\alpha-2}(\nabla u)^{2}\right. \\
& +(\beta(d-2)-k) \int u^{\alpha-4 / d} R^{(d)}+\int u^{\alpha}\left(2 \beta T^{(d)}-2 T^{(k)}\right) .
\end{aligned}
$$

To get the combination in eq. (2.6), we take $\beta=k /(d-2)$. Multiplying by $-(d-$ $2) / 2(d+k-2)$, we obtain

$$
\int u^{\alpha} R^{(k)}=-2\left(\alpha-\frac{2}{d}\right) \int u^{\alpha-2}(\nabla u)^{2}+\frac{1}{d+k-2} \int u^{\alpha}\left((d-2) T^{(k)}-k T^{(d)}\right) .
$$

Taking $\alpha=2 / d$, the term involving derivatives of the warp factor drops out, to give an integral version of eq. (2.6) directly analogous to that in the no go theorem of [9-12]. Thus, warping alone will not allow a supergravity solution with everywhere negative curvature. 
On the other hand, this integrated type of no go theorem can be violated in string/M theory by localized sources such as $\mathrm{O} 6$ planes.

To get a condition which applies at each point of $M$, one can find another linear combination of the $R_{4}$ and $R_{6}$ equations in which the warping contribution is non-positive definite. This is equation (5.6) in [16], which in these dimensions is

$$
R_{6}=-12(\nabla A)^{2}+e^{-2 A} R_{4}+\frac{3}{4} T_{6}-\frac{5}{4} T_{4} .
$$

While the combination of $T_{6}$ and $T_{4}$ appearing here is different from that in eq. (2.5), one can check that it too is always positive for fluxes. Thus, one sees that, at least for de Sitter and Minkowski space-times, the same conclusion would apply (ruling out negative scalar curvature) without warping, but warping provides a negative contribution to the scalar curvature.

Physically, by the discussion in section 3 of [16], the total vacuum energy is an integral of the local energy densities weighted by the warp factor. Since the warp factor is large in regions of negative curvature and positive energy, and small in regions of positive curvature and negative energy, this weighing will also tend to increase the total vacuum energy and thus favor de Sitter. This weighing (by $u^{2}$ ) is different from that in eq. (3.6), so it is still possible that negative curvature is relevant for this.

\subsection{Conformal factor}

We now consider an internal metric

$$
\tilde{g}_{i j}=e^{2 B} g_{i j}
$$

where $B$ is a function on the compactification manifold. The formula expressing the curvature $\tilde{R}_{6}$ of $\tilde{g}$, in terms of $R_{6}$ and $B$, is standard (e.g. see the appendix of Wald's textbook):

$$
\tilde{R}_{6}=e^{-2 B}\left(R_{6}-10 \nabla^{2} B-20(\nabla B)^{2}\right) .
$$

Locally, this can modify $R_{6}$ in either direction (more positive or more negative).

Since the extra terms are not a total derivative, there is no topological constraint on the integrated scalar curvature (in greater than two dimensions), and varying the conformal factor will change the integrated scalar curvature. The overall change will tend to be positive. This can be seen by computing

$$
\begin{aligned}
\int \sqrt{\tilde{g}} \tilde{R}_{6} & =\int \sqrt{g} e^{4 B}\left(R_{6}-10 \nabla^{2} B-20(\nabla B)^{2}\right) \\
& =\int \sqrt{g} e^{4 B}\left(R_{6}+20(\nabla B)^{2}\right)
\end{aligned}
$$

after an integration by parts. This conclusion can be strengthened by making the change of variables

$$
v=e^{2 B},
$$

in terms of which

$$
\int \sqrt{\tilde{g}} \tilde{R}_{6}=\int \sqrt{g}\left(5(\nabla v)^{2}+v^{2} R_{6}\right)
$$


We can regard this as a functional of the conformal factor $v$, then there is a global constraint - if we set the overall scale of the metric, say by constraining the total volume, then the integrated scalar curvature has a definite minimum value. This is the subject of the Yamabe problem in mathematics [27]. The minimum tends to be realized by the constant curvature metric, while a nonconstant conformal factor will increase the integrated scalar curvature.

There are topological constraints on the manifolds which can realize metrics with positive scalar curvature at every point, and even on those which can have non-negative scalar curvature at every point [1]. However, if we weaken this to allow negative scalar curvature at even a single point, in $k \geq 3$ there is no constraint [29]. A more physics-friendly explanation of this point can be found in [30].

The conformal factor is used in well known solutions such as [24] and the GKP vacua [17]. These are flux compactifications on Calabi-Yau manifolds, and one might worry about the same type of contradiction: the flux is a positive source to eq. (2.27), but of course a Calabi-Yau manifold has $R_{6}=0$. The resolution is that these metrics are only conformally Calabi-Yau.

Taking this into account, we can imagine finding a IIa solution in which the curvature is negative near the $\mathrm{O} 6$ planes, and positive elsewhere. A good example is the IIa solution of [31], which is obtained by T-duality from a GKP flux solution on a torus. Of course, since this will increase the total integrated scalar curvature, this would be expected to reduce or eliminate the effect of negative curvature in favoring de Sitter space-time. In the Calabi-Yau solutions, arguments using supersymmetry and no-scale structure showed that the solutions remained Minkowski (at least, before fixing Kähler moduli and uplifting). At present we have no analog of these arguments for de Sitter solutions.

\section{Discussion}

Except for a few very symmetric examples, finding explicit compactifications of supergravity by solving the equations of motion is very difficult. After a great deal of study, there are more or less two successful approaches.

One is to appeal to mathematical existence theorems showing that certain solutions exist, and then perturb around these. This is how the original Calabi-Yau compactifications of the heterotic string were justified, and barring the discovery of exact metrics on Calabi-Yau or other manifolds leading to quasi-realistic compactifications, is likely to remain the "gold standard." From this point of view, further development of a more mathematical approach to string compactification, as illustrated for example by [32], would be very helpful.

The other approach is to assume that the problem is governed by a lower dimensional effective theory, construct its effective potential, and argue that it has a local minimum. In fact one can think of the solution of supergravity equations of motion as minimizing an effective potential $[16,33]$, so the new assumption here is that one can reduce this to minimizing with respect to a finite number of fields. The justification for this [34] is that one can solve the equations of motion for massive fields in a perturbative series, schematically

$$
M^{2} \phi^{i}=J^{i}-g_{j k}^{i} \phi^{j} \phi^{k}+\ldots
$$


The meaning of this expression is as follows. Suppose one has a "near-solution" which approximately solves the equations of motion; one can try to develop a Kaluza-Klein expansion around it, in which variations of the massive fields are denoted $\phi^{i}$. Since it is not a solution, the sources $J^{i}$ will be non-zero, but if they are small, one can develop a series expansion in powers of $g J / M^{2}$ for the exact solution. In classical field theory, such expansions often have finite radius of convergence.

The effective action approach is simpler and in many ways more physical, as it focuses attention on the relevant variables. As outlined at the end of section 2.0.1, one can partially understand the no-go theorem given here in these terms - both flux and negative curvature sources of energy fall off too rapidly at large volume to allow a local minimum. ${ }^{2}$

This interpretation of the no-go theorem suggests that one can get around it by incorporating additional stringy effects in the potential. While this may be, the need to satisfy the Einstein equation at every point in the internal manifold suggests potential pitfalls with this. One is that one can leave out a field which becomes tachyonic at the solution of interest. In the absence of arguments such as supersymmetry, this could become more likely as one moves away from a known higher dimensional solution.

A second potential pitfall is that the actual vacuum with massive modes may be qualitatively different in some way from the original near-solution. In the present case of constructing de Sitter, there are at least two ways this could happen:

1. As one turns on massive fields, the value of the potential will decrease. This follows from standard variational arguments, or could be thought of as following from a "flow" down the potential gradient. Thus, even if the original value of the potential is positive, the value at the true solution could be negative.

2. Symmetries or near-symmetries of the near-solution might be broken in this process. For example, a shift symmetry which would tell us that the effective potential is flat in some direction, might be broken.

In fact, because of the argument we just gave that one needs the warp and/or conformal factors to construct a negative curvature solution, both possibilities would seem potentially relevant. Any nontrivial warp or conformal factor will by definition break translational symmetries on $M$. And, the effective potential is unbounded below under generic variations of these factors not satisfying the constraints. Even with the constraint, there may be a lot of room to decrease the potential.

It seems very important to find some higher dimensional solutions, at least as explicit as [17] gave for IIb flux vacua, to evaluate these points.

\section{Acknowledgments}

We thank E. Bergshoeff, X.X. Chen, M. Hertzberg, S. Kachru, A. Linde, D. Lust, B. McInnes, G. Moore, D. Roest, E. Silverstein, G. Shiu, A. Van Proeyen and A. Westphal for useful discussions. We also thank I. Arefeva, J. Russo and P. Townsend for stressing

\footnotetext{
${ }^{2}$ One can also understand time-dependent solutions with negative curvature, such as [35], in these terms — kinetic energy allows rolling up the potential. [36]
} 
to us that the arguments here apply only to maximally symmetric space-times. The work of MRD is supported by DOE grant DE-FG02-92ER40697, and the work of RK is supported by NSF grant 0756174 .

\section{A Type IIA, IIB $d=10$ Supergravity Energy-Momentum Tensor}

Here we review the democratic version of $d=10$ supergravity [14, 15], in which it is manifest that the stress-tensors for form fields is quadratic. It also has the virtue of treating the IIA, IIB, and massive IIA cases in a unified way.

The idea is to write a "pseudo-action," which is a functional of both the R-R potentials and their duals. To remove this doubling of the number of degrees of freedom, one imposes the duality relations as additional constraints, which do not follow from varying the pseudoaction. The combined system of variational equations and constraints is equivalent to the standard equations of motion of the theory.

The pseudo-action has the extended field content

$$
\begin{array}{ll}
\text { IIA : } & \left\{g_{\mu \nu}, B_{\mu \nu}, \phi, C_{\mu}^{(1)}, C_{\mu \nu \rho}^{(3)}, C_{\mu \cdots \rho}^{(5)}, C_{\mu \cdots \rho}^{(7)}, C_{\mu \cdots \rho}^{(9)}, \psi_{\mu}, \lambda\right\}, \\
\text { IIB : } & \left\{g_{\mu \nu}, B_{\mu \nu}, \phi, C^{(0)}, C_{\mu \nu}^{(2)}, C_{\mu \cdots \rho}^{(4)}, C_{\mu \cdots \rho}^{(6)}, C_{\mu \cdots \rho}^{(8)}, \psi_{\mu}, \lambda\right\} .
\end{array}
$$

It is understood that in the IIA case the fermions contain both chiralities, while in the IIB case they satisfy $\Gamma_{11} \psi_{\mu}=\psi_{\mu}, \Gamma_{11} \lambda=-\lambda$. The bosonic part of the pseudo-action in Einstein frame is

$$
S=-\frac{1}{2} \int d^{10} x \sqrt{-g}\left[R(\omega(e))+\frac{1}{2}(\partial \phi)^{2}+\frac{1}{2} e^{-\phi} H \cdot H\right]+\sum_{n=0,1 / 2}^{5,9 / 2} \frac{1}{4} e^{(5-2 n) \phi / 2} G^{(2 n)} \cdot G^{(2 n)} .
$$

The summation in the above pseudo-action is over integers $(n=0,1, \ldots, 5)$ in the massive IIA case, over the integers $(n=1, \ldots, 4)$ for standard IIA, and over half-integers $(n=$ $1 / 2,3 / 2, \ldots, 9 / 2)$ for IIB. In the summation range we will always first indicate the lowest value for the IIA case, before the one for the IIB case. For notational convenience we group all potentials and field strengths in the formal sums

$$
\mathbf{G}=\sum_{n=0,1 / 2}^{5,9 / 2} G^{(2 n)}, \quad \mathbf{C}=\sum_{n=1,1 / 2}^{5,9 / 2} C^{(2 n-1)} .
$$

where $G^{p} \equiv \frac{1}{p !} G_{M_{1} \ldots M_{p}} d x^{M_{1}} \wedge \ldots \wedge d x^{M_{p}}$ and $C^{q} \equiv \frac{1}{q !} C_{M_{1} \ldots M_{q}} d x^{M_{1}} \wedge \ldots \wedge d x^{M_{q}}$. Here the meaning of each term in the sum in the action is

$$
G^{(p)} \cdot G^{(p)} \equiv \frac{1}{p !} G_{M_{1} \ldots M_{p}} G_{N_{1} \ldots N_{p}} g^{M_{1} N_{1}} \ldots g^{M_{p} N_{p}}
$$

The bosonic field strengths are given by

$$
H=d B, \quad \mathbf{G}=d \mathbf{C}-d B \wedge \mathbf{C}+G^{(0)} \mathbf{e}^{B},
$$


where it is understood that each equation involves only one term from the formal sums (A.3) (only the relevant combinations are extracted). The corresponding Bianchi identities then read $d H=0, d \mathbf{G}-H_{\wedge} \mathbf{G}=0$. Here $G^{(0)}=m$ is the constant mass parameter of IIA supergravity. In the IIB theory all equations should be read with vanishing $G^{(0)}$.

Due to the appearance of all R-R potentials, the number of degrees of freedom in the $\mathrm{R}-\mathrm{R}$ sector has been doubled. To get the correct number of degrees of freedom, we impose the duality relations in the bosonic sector of the theory which in the Einstein frame reads

$$
G^{(2 n)}=(-)^{\operatorname{Int}[n]} e^{(2 n-5) \phi / 2} \star G^{(10-2 n)},
$$

These duality relations are combined with the equations of motion that follow from the pseudo-action (A.2).

Our purpose here is to identify the energy-momentum tensor for all $d=10$ supergravity fields which in the Einstein frame defines the right hand side of the Einstein tensor. For the Einstein equations the only thing that matters is the tensor character of the flux, how many contractions $g^{M N}$ are there. The fact that the flux is multiplied by a function of the dilaton does not matter. It matters that the terms proportional to $(\partial \phi)^{2}, H \cdot H$ and $G^{(2 n)} \cdot G^{(2 n)}$ all have the same sign as one can see from the action (A.2). This means that the contribution to $T_{M N}$ from all $d=10$ supergravities comes from a class of energymomentum tensors quadratic in antisymmetric $p$-rank tensors $F_{M_{1}, \ldots M_{p}}$ where $p=0, . ., 9$ which all have positive sign in the Einstein frame. This forms a basis for the detailed computations in section 2 .

Open Access. This article is distributed under the terms of the Creative Commons Attribution Noncommercial License which permits any noncommercial use, distribution, and reproduction in any medium, provided the original author(s) and source are credited.

\section{References}

[1] M. Berger, A panoramic view of Riemannian geometry, Springer-Verlag, Berlin Germany (2003).

[2] E. Silverstein, Simple de Sitter solutions, Phys. Rev. D 77 (2008) 106006 [arXiv:0712.1196] [SPIRES].

[3] L.J. Romans, Massive $N=2 a$ supergravity in ten-dimensions, Phys. Lett. B 169 (1986) 374 [SPIRES].

[4] O. DeWolfe, A. Giryavets, S. Kachru and W. Taylor, Type IIA moduli stabilization, JHEP 07 (2005) 066 [hep-th/0505160] [SPIRES].

[5] R. Kallosh and M. Soroush, Issues in type IIA uplifting, JHEP 06 (2007) 041 [hep-th/0612057] [SPIRES].

[6] M.P. Hertzberg, S. Kachru, W. Taylor and M. Tegmark, Inflationary Constraints on Type IIA String Theory, JHEP 12 (2007) 095 [arXiv:0711.2512] [SPIRES].

[7] E. Silverstein and A. Westphal, Monodromy in the CMB: gravity waves and string inflation, Phys. Rev. D 78 (2008) 106003 [arXiv:0803.3085] [SPIRES]. 
[8] B. Greene, D. Kabat, J. Levin and D. Thurston, A bulk inflaton from large volume extra dimensions, arXiv: 1001.1423 [SPIRES].

[9] G.W. Gibbons, Aspects of supergravity theories, lecture given at GIFT Seminar on Theoretical Physics, San Feliu de Guixols, Spain, Jun 4-11, 1984, published in GIFT Seminar 1984:0123 (QCD161:G2:1984) [SPIRES].

[10] G.W. Gibbons, Thoughts on tachyon cosmology, Class. Quant. Grav. 20 (2003) S321 [hep-th/0301117] [SPIRES].

[11] B. de Wit, D.J. Smit and N.D. Hari Dass, Residual supersymmetry of compactified D $=10$ supergravity, Nucl. Phys. B 283 (1987) 165 [SPIRES].

[12] J.M. Maldacena and C. Núnez, Supergravity description of field theories on curved manifolds and a no go theorem, Int. J. Mod. Phys. A 16 (2001) 822 [hep-th/0007018] [SPIRES].

[13] R.M. Wald, General relativity, University of Chicago Press, Chicago U.S.A. (1984).

[14] E. Bergshoeff, R. Kallosh, T. Ortín, D. Roest and A. Van Proeyen, New formulations of $D=10$ supersymmetry and D8-O8 domain walls, Class. Quant. Grav. 18 (2001) 3359 [hep-th/0103233] [SPIRES].

[15] D. Roest, M-theory and gauged supergravities, Fortsch. Phys. 53 (2005) 119 [hep-th/0408175] [SPIRES].

[16] M.R. Douglas, Effective potential and warp factor dynamics, JHEP 03 (2010) 071 [arXiv:0911.3378] [SPIRES].

[17] S.B. Giddings, S. Kachru and J. Polchinski, Hierarchies from fluxes in string compactifications, Phys. Rev. D 66 (2002) 106006 [hep-th/0105097] [SPIRES].

[18] S.B. Giddings, The fate of four dimensions, Phys. Rev. D 68 (2003) 026006 [hep-th/0303031] [SPIRES].

[19] B.S. Acharya, F. Benini and R. Valandro, Fixing moduli in exact type IIA flux vacua, JHEP 02 (2007) 018 [hep-th/0607223] [SPIRES].

[20] C. Caviezel et al., The effective theory of type IIA AdS $S_{4}$ compactifications on nilmanifolds and cosets, Class. Quant. Grav. 26 (2009) 025014 [arXiv:0806.3458] [SPIRES].

[21] U.H. Danielsson, S.S. Haque, G. Shiu and T. Van Riet, Towards classical de Sitter solutions in string theory, JHEP 09 (2009) 114 [arXiv: 0907.2041] [SPIRES].

[22] B.S. Acharya and M.R. Douglas, A finite landscape?, hep-th/0606212 [SPIRES].

[23] K. Becker, M. Becker, M. Haack and J. Louis, Supersymmetry breaking and $\alpha^{\prime}$-corrections to flux induced potentials, JHEP 06 (2002) 060 [hep-th/0204254] [SPIRES].

[24] K. Becker and M. Becker, M-theory on eight-manifolds, Nucl. Phys. B 477 (1996) 155 [hep-th/9605053] [SPIRES].

[25] V. Balasubramanian, P. Berglund, J.P. Conlon and F. Quevedo, Systematics of moduli stabilisation in Calabi-Yau flux compactifications, JHEP 03 (2005) 007 [hep-th/0502058] [SPIRES].

[26] J.P. Conlon, F. Quevedo and K. Suruliz, Large-volume flux compactifications: moduli spectrum and D3/D7 soft supersymmetry breaking, JHEP 08 (2005) 007 [hep-th/0505076] [SPIRES]. 
[27] J.M. Lee and T.H. Parker, The Yamabe problem, Bull. Amer. Math. Soc. (N.S.) 17 (1987) 1.

[28] J. Petean, The Yamabe invariant of simply connected manifolds, J. Reine Angew. Math. 523 (2000) 225 [math/9808062].

[29] J.L. Kazdan and F.W. Warner, Existence and conformal deformation of metrics with prescribed Gaussian and scalar curvatures, Ann. Math. 101 (1975) 317.

[30] B. McInnes, The Arrow Of Time In The Landscape, arXiv:0711.1656 [SPIRES].

[31] S. Kachru, M.B. Schulz, P.K. Tripathy and S.P. Trivedi, New supersymmetric string compactifications, JHEP 03 (2003) 061 [hep-th/0211182] [SPIRES].

[32] J.-X. Fu and S.-T. Yau, The theory of superstring with flux on non-Kähler manifolds and the complex Monge-Ampere equation, J. Diff. Geom. 78 (2009) 369 [hep-th/0604063] [SPIRES].

[33] S.B. Giddings and A. Maharana, Dynamics of warped compactifications and the shape of the warped landscape, Phys. Rev. D 73 (2006) 126003 [hep-th/0507158] [SPIRES].

[34] E. Witten, New issues in manifolds of SU(3) holonomy, Nucl. Phys. B 268 (1986) 79 [SPIRES].

[35] P.K. Townsend and M.N.R. Wohlfarth, Accelerating cosmologies from compactification, Phys. Rev. Lett. 91 (2003) 061302 [hep-th/0303097] [SPIRES].

[36] R. Emparan and J. Garriga, A note on accelerating cosmologies from compactifications and S-branes, JHEP 05 (2003) 028 [hep-th/0304124] [SPIRES]. 